11

\title{
Радиолокационные измерения амплитуды вибрации
}

\author{
() А.С. Запевалов, ${ }^{1}$ А.Н. Пинчук, ${ }^{2}$ В.М. Бурдюгов ${ }^{1}$ \\ ${ }^{1}$ Морской гидрофизический институт РАН, \\ 299011 Севастополь, Россия \\ 2Черноморское высшее военно-морское училище им. П.С. Нахимова, \\ 299000 Севастополь, Россия \\ e-mail: sevzepter@mail.ru
}

(Поступило в Редакцию 16 января 2018 г.)

Предложен метод измерения амплитуды гармонической вибрации отражателя с помощью когерентной радиолокационной станции. Построена математическая модель, описывающая зависимость доплеровского спектра отраженного радиосигнала от соотношения амплитуды вибрации и длины радиоволны. Расчет амплитуды вибрации осуществляется по отношению амплитуд третьей и первой или четвертой и второй гармоник.

DOI: $10.21883 / J T F .2018 .12 .46794 .27-18$

Методы определения вибрации можно разделить на две основные группы: контактные, при которых датчик имеет механическую связь с исследуемым объектом, и дистанционные, при которых эта связь отсутствует. Для дистанционного определения параметров вибрации используются оптические, акустические и радиоволновые методы [1].

В последние годы широкое распространение получили оптические методы определения вибрации, основанные на лазерном зондировании вибрирующей поверхности [2,3]. Их общим недостатком является сложность и высокая стоимость оборудования, а также строгие требования к качеству поверхности исследуемого объекта. Как правило, оптические методы используются в лабораторных условиях.

Акустический метод (ультразвуковая фазометрия) заключается в измерении разности фаз опорного сигнала ультразвуковой частоты и сигнала, отраженного от исследуемого объекта. Использование этого метода ограничивает невысокая разрешающая способность, сильное затухание ультразвука в воздухе, зависимость точности измерения от частоты вибрации [4]. Акустический метод, как и оптический метод, в основном применяется при лабораторных измерениях.

Наибольшее распространение среди радиоволновых методов измерения вибраций получили интерференционные методы, основанные на облучении вибрирующей поверхности радиоволнами СВЧ диапазона. Эти методы в первую очередь предназначены для измерений в полевых условиях [5,6]. Выделенную часть зондирующего сигнала радиолокационной станции (РЛС) используют в качестве когерентного сигнала и смешивают ее с сигналом, отраженным от вибрирующей поверхности. Смешанный сигнал трансформируют в сигнал промежуточной частоты, который усиливают, осуществляют фазовое детектирование для формирования видеосигнала, усиливают его и выделяют из него сигнал доплеровской частоты. У выделенного сигнала амплитуда пропорци- ональна амплитуде вибраций, а частота соответствует частоте вибраций контролируемого объекта. Однако прямое измерение амплитуды вибрации, проводимое по амплитуде выделенного сигнала, требует выполнения сложных процедур калибровки, которая меняется при изменении расстояния от РЛС до контролируемого объекта [7].

Целью настоящей работы является обоснование возможности измерения амплитуды вибрации на основе трансформации доплеровского спектра при радиозондировании вибрирующей поверхности когерентной РЛС.

Построим спектральную модель сигнала, отраженного от вибрирующей поверхности. Рассмотрим ситуацию, при которой источник когерентного непрерывного излучения облучает вибрирующую плоскую поверхность. Вибрация приводит к смещению $\xi(t)$ вибрирующей поверхности в направлении источника излучения, которое соответствует гармоническому закону

$$
\xi(t)=\xi_{0} \sin \left(\Omega_{0} t\right),
$$

где $\xi_{0}-$ амплитуда вибрации; $\Omega_{0}$ - частота вибрации; $t$ - время. Примем, что амплитуда зондирующего сигнала равна $A_{0}$, несущая частота - $\omega_{0}$, и представим его в форме $S_{0}(t)=A_{0} \cos \left(\omega_{0} t\right)$. Расстояние $r(t)$ между источником излучения и отражающей поверхностью отклоняется от среднего $r_{0}$, меняясь во времени как $r(t)=r_{0}+\xi_{0} \sin \left(\Omega_{0} t\right)$.

В этом случае отраженный сигнал можно представить в виде

$$
S(t)=A_{0} \cos \left[\left(\omega_{0} t\right)-\frac{4 \pi \xi_{0}}{\lambda} \sin \left(\Omega_{0} t\right)-\frac{4 \pi r_{0}}{\lambda}+\varphi_{0}\right],
$$

где $\lambda$ - длина радиоволны, $\varphi_{0}-$ изменение фазы сигнала при отражении. Всю информацию об амплитуде и частоте вибрации несет в себе изменение фазы отраженного сигнала $\left(4 \pi \xi_{0} / \lambda\right) \sin (\Omega t)$. Изменение фазы происходит с частотой вибрации. Амплитуда изменения фазы прямо пропорциональна амплитуде вибрации. 

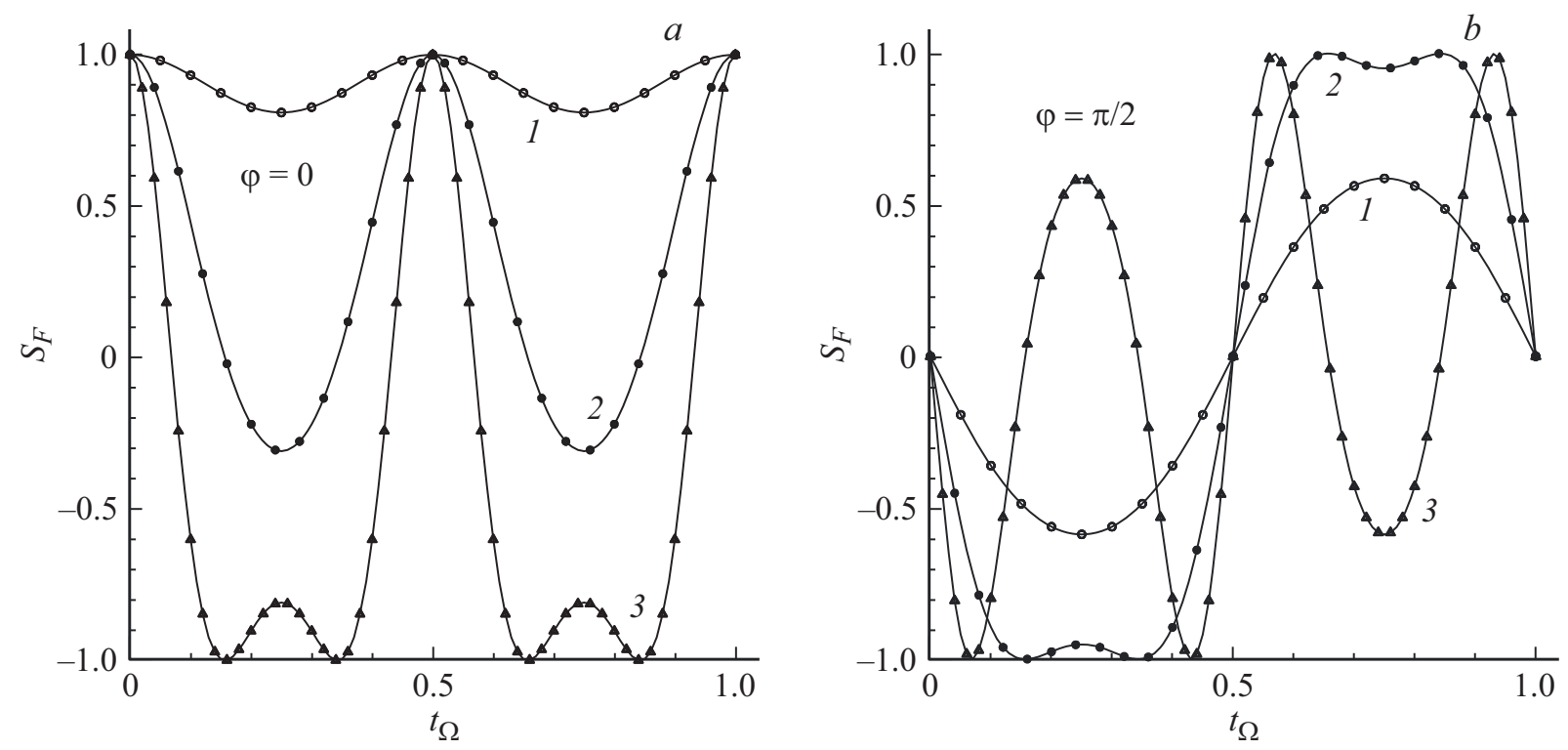

Рис. 1. Вид сигнала $S_{F}=S_{F}\left(t_{\Omega}\right)$ для $\varphi=0$ и $\varphi=\pi / 2$. Кривые $1-3$ соответствуют $0.1,0.3$ и 0.6 соответственно.

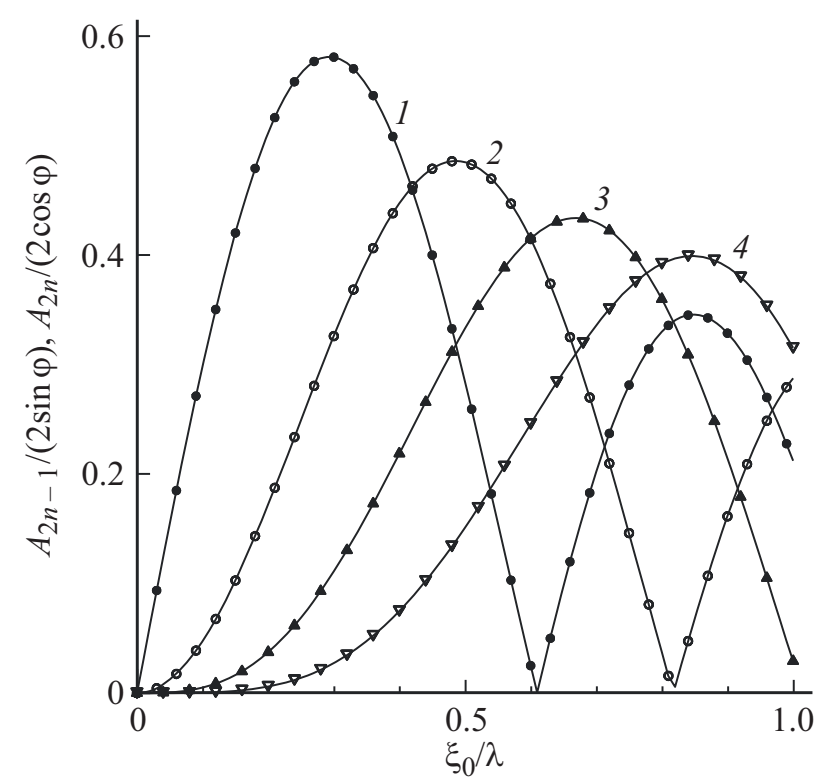

Рис. 2. Зависимости амплитуд гармоник $A_{n}$ от отношения $\xi_{0} \lambda$. Кривые 1-4 соответствуют первым четырем гармоникам.

После фазового детектирования сигнала $S(t)$ получаем

$$
S_{F}(t)=A_{F} \cos [m \sin (\Omega t)+\varphi],
$$

где $A_{F}-$ амплитуда, $m=4 \pi \xi_{0} / \lambda$ индекс фазовой модуляции, $\varphi=-4 \pi r_{0} / \lambda+\varphi_{0}$. Выражение (1) является нелинейным. Вид спектра $S_{F}(t)$ сигнала зависит от индекса фазовой модуляции, что, в принципе, позволяет решать обратную задачу: определять амплитуду вибрации по спектру сигнала $S_{F}(t)$.

Разложим функцию в ряд Фурье. После ряда простых, но громоздких преобразований, которые здесь не приво- дятся, получаем следующее выражение [6]:

$$
\begin{aligned}
S_{f}(t) & =A_{\mathrm{F}}\left[J_{0}(m)+2 \cos \varphi \sum_{n=1}^{\infty} J_{2 n}(m) \cos (2 n \Omega t)\right. \\
& \left.-2 \sin \varphi \sum_{n=1}^{\infty} J_{2 n-1}(m) \cos ((2 n-1) \Omega t),\right]
\end{aligned}
$$

где $J_{n}(z)=\pi^{-1} \int_{0}^{\pi} \cos (n \tau-z \sin \tau) d \tau-$ функции Бесселя первого рода порядка $n$. В случае, когда $\varphi \equiv 0$ или $\varphi \equiv \pi$, сигнал $S_{F}(t)$ содержит только четные гармоники, когда $\varphi \equiv \pi / 2$ или $\varphi \equiv 3 \pi / 2-$ только нечетные гармоники. Спектр сигнала является дискретной функцией частоты, на частотах, кратных частоте вибрации, его значения пропорциональны $\left(\cos \varphi J_{2 n}(m)\right)^{2}$ или $\left(\sin \varphi J_{2 n-1}(m)\right)^{2}$.

В общем случае, как следует из (2), соотношение между амплитудами нечетных и четных гармоник определяется индексом фазовой модуляции $m$ и фазовым сдвигом $\varphi$, который, в свою очередь, зависит от среднего расстояния между локатором и вибрирующей поверхностью. Отношения амплитуд четных гармоник, а также отношения амплитуд нечетных гармоник от фазового сдвига $\varphi$ не зависят и определяются только индексом фазовой модуляции. Вид сигнала $S_{F}(t)$ показан на рис. 1. При построении графиков введено относительное время $t_{\Omega}=t / T_{\Omega}$ (здесь $T_{\Omega}-$ период вибрации). Принято, что $A_{F}=1$. Расчеты проведены для трех значений индекса фазовой модуляции, которые соответствуют трем значениям отношения амплитуды вибрации и длины радиоволны $\xi_{0} / \lambda=01,0.3$ и 0.6 .

В частном случае может возникнуть ситуация, когда расстояние от вибрирующей поверхности до РЛС изменяется таким образом, что фазовый сдвиг $\varphi$ медленно 


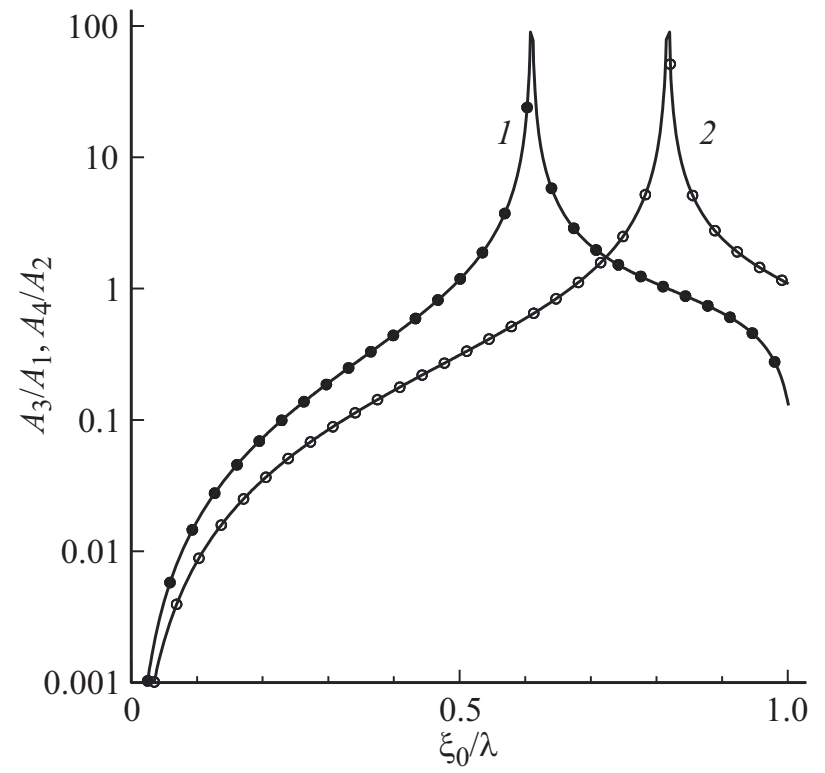

Рис. 3. Зависимости отношений амплитуд гармоник вибрации $A-3 / A_{1}(1)$ и $A_{4} / A_{2}(2)$ от отношения $\xi / \lambda$.

(по сравнению с периодом вибрации) меняется и равномерно распределен на интервале от 0 до $2 \pi$. В этом случае отношения амплитуд гармоник на основной частоте вибрации и на кратных ей частотах будут определяться только одним параметром - индексом фазовой модуляции. Соответственно амплитуду вибрации можно определить по отношению амплитуд второй и первой гармоник.

Чтобы оценить изменения вклада отдельных гармоник в сигнал $S_{F}(t)$, рассмотрим ситуацию, когда $\varphi=\pi / 4$. В этом случае амплитуды гармоник с точностью до постоянного для всех гармоник множителя равны $A_{n}=J_{n}(m)$. Изменения амплитуды $A_{n}$ при изменении отношения $\xi_{0} / \lambda$ показаны на рис. 2 . С ростом отношения $\xi_{0} / \lambda$ вклад старших гармоник растет, причем амплитуды гармоник меняются немонотонно.

Немонотонный характер изменения параметров $A_{n}$ накладывает ограничения на возможность определения амплитуды вибрации путем радиолокационных измерений с помощью когерентной РЛС. Ограничения вызваны тем, что разным значениям отношения $\xi_{0} / \lambda$ могут соответствовать одни и те же значения отношений амплитуд четных и нечетных гармоник. Как меняются отношения амплитуд гармоник $A_{3} / A_{1}$ и $A_{4} / A_{2}$ от отношения $\xi_{0} / \lambda$, показано на рис. 3. При $\xi_{0} / \lambda=0.614$ амплитуда $A_{1}$ принимает нулевое значение, соответственно отношение $A_{3} / A_{1}$ обращается в бесконечность. Амплитуда $A_{2}=0$ при $\xi_{0} / \lambda=0.817$. Отсюда следует, что рассмотренный в настоящей работе метод измерения амплитуды вибрации ограничен сверху максимальным значением амплитуды $\xi_{\text {max }}=0.614 \lambda$.

Пороговое значение или нижняя граница измерения амплитуды вибрации $\xi_{\min }$ определяется собственными шумами когерентной РЛС. Кроме того, при измерениях в полевых условиях на величину $\xi_{\min }$ могут влиять отражения от фоновых элементов, если эти элементы являются подвижными. В частности, при проведении измерений, в рамках которых определяется вибрация нефтяных и океанографических платформ или корпуса судна, значительный вклад в регистрируемый сигнал дает рассеяние радиоволн на морской поверхности [8].

Таким образом, показана возможность определения амплитуды вибрации путем радиолокационного зондирования вибрирующей поверхности когерентной РЛС. В основе предложенного метода лежит нелинейная трансформация доплеровского спектра радиосигнала, отраженного от гармонически вибрирующей поверхности при изменении ее амплитуды. Амплитуда вибрации рассчитывается по отношению амплитуд первой и третьей гармоник или отношению амплитуд второй и четвертой гармоник.

Работа выполнена в рамках проекта Морского гидрофизического института РАН тема № 0827-2014-0011.

\section{Список литературы}

[1] Волковеи, А.И., Руденко Д.Ф., Гусинский А.В., Кострикин А.М. // Доклады БГУИР. 2007. № 4 (20). С. 58-64.

[2] Усанов Д.А., Скрипаль А.В., Астахов Е.И. // ЖТФ. 2013. Т. 83. Вып. 12. С. 152-154.

[3] Кочегаров И.И., Моисеев С.А. // Труды междунар. симпозиума Надежность и качество. 2015. Т. 2. С. 301-303.

[4] Гордеев Б.А., Новожилов М.В., Образцов Д.И. // Метрология. 1990. № 6. С. 33-36.

[5] Андреев Г.А., Хохлов Г.И. // Электромагнитные волны и электронные системы. 1997. Т. 2. № 4. С. 99-103.

[6] Пинчук А.Н. // Наука и образование. 2014. № 6. С. 270-278. DOI:10.7463/0614.0715325

[7] Викторов В.А., Лункин Б.В., Совлуков А.С. Радиоволновые измерения параметров технологических процессов. М.: Энергоатомиздат, 1989. С. 124-162.

[8] Запевалов А.С. // Известия РАН. Физика атмосферы и океана. 2009. Т. 45. o 2. С. 266-275. [Zapevalov A.S. // Izvestiya-Atmospheric and Ocean Physics. 2009. Vol. 45. N 2. P. 253-261. 